\title{
Impact of schizophrenia genetic liability on the association between schizophrenia and physical illness: data-linkage study
}

Kimberley M. Kendall, Ann John, Sze Chim Lee, Elliott Rees, Antonio F. Pardiñas, Marcos Del Pozo Banos, Michael J. Owen, Michael C. O’Donovan, George Kirov, Keith Lloyd, lan Jones, Sophie E. Legge* and James T. R. Walters*

\section{Background}

Individuals with schizophrenia are at higher risk of physical illnesses, which are a major contributor to their 20-year reduced life expectancy. It is currently unknown what causes the increased risk of physical illness in schizophrenia.

\section{Aims}

To link genetic data from a clinically ascertained sample of individuals with schizophrenia to anonymised National Health Service (NHS) records. To assess (a) rates of physical illness in those with schizophrenia, and (b) whether physical illness in schizophrenia is associated with genetic liability.

\section{Method}

We linked genetic data from a clinically ascertained sample of individuals with schizophrenia (Cardiff Cognition in Schizophrenia participants, $n=896$ ) to anonymised NHS records held in the Secure Anonymised Information Linkage (SAIL) databank. Physical illnesses were defined from the General Practice Database and Patient Episode Database for Wales. Genetic liability for schizophrenia was indexed by (a) rare copy number variants (CNVs), and (b) polygenic risk scores.

\section{Results}

Individuals with schizophrenia in SAIL had increased rates of epilepsy (standardised rate ratio $(S R R)=5.34$ ), intellectual disability (SRR $=3.11$ ), type 2 diabetes $(S R R=2.45)$, congenital disorders $(S R R=1.77)$, ischaemic heart disease $(S R R=1.57)$ and smoking $(S R R=1.44)$ in comparison with the general SAlL population. In those with schizophrenia, carrier status for schizophrenia-associated CNVs and neurodevelopmental disorder-associated CNVs was associated with height $(P=0.015-0.017)$, with carriers being 7.5-7.7 $\mathrm{cm}$ shorter than non-carriers. We did not find evidence that the increased rates of poor physical health outcomes in schizophrenia were associated with genetic liability for the disorder.

\section{Conclusions}

This study demonstrates the value of and potential for linking genetic data from clinically ascertained research studies to anonymised health records. The increased risk for physical illness in schizophrenia is not caused by genetic liability for the disorder.

\section{Keywords}

Schizophrenia; psychotic disorders; genetics; physical health.

\section{Copyright and usage}

(C) The Author(s) 2020. Published by Cambridge University Press on behalf of the Royal College of Psychiatrists. This is an Open Access article, distributed under the terms of the Creative Commons Attribution licence (http://creativecommons.org/ licenses/by/4.0/), which permits unrestricted re-use, distribution, and reproduction in any medium, provided the original work is properly cited.

\section{Background}

Individuals with schizophrenia have a 20 -year reduced life expectancy. ${ }^{1}$ A major contributing factor is the increased rate of poor physical health outcomes in individuals with schizophrenia, related to conditions such as metabolic, cardiovascular and respiratory disease. ${ }^{2}$ Identifying the underlying reasons for these health disparities will provide a first step towards closing this health gap and thus has become a priority in schizophrenia research and clinical care in recent years. ${ }^{3}$ It is unclear whether the poorer physical health outcomes in schizophrenia (a) arise from the pleiotropic action of schizophrenia risk factors or (b) are secondary to illnessrelated factors such as negative symptoms, adverse effects of treatment (particularly antipsychotic medication) or poorer access to healthcare.

Genetic factors make major contributions to schizophrenia risk, arising from both common genetic variation and rare variants such as copy number variants (CNVs), the latter of these also being associated with physical health consequences in population samples. ${ }^{4,5}$ A recent study, in which genetic and electronic health record data were linked for 106160 individuals, reported associations between polygenic risk scores for schizophrenia and several physical health

* Joint last authors. phenotypes including smoking and reduced rates of obesity. ${ }^{6}$ At present, it is not known whether these genetic risk factors for schizophrenia are also associated with physical ill health in those with the disorder.

One reason for the dearth of research examining genetics and physical health outcomes in those with mental health disorders is likely to be the lack of available physical health data in existing psychiatric cohorts. In contrast, linkage of national registry data to anonymised mental and physical healthcare records, as exemplified in Nordic countries, has provided a rich resource for mental health research and led to important insights including into physical health outcomes in schizophrenia. ${ }^{7,8}$ The use of anonymised National Health Service (NHS) records and linkage of patient data to national registries in the UK is gaining momentum ${ }^{9,10}$ and this approach has enabled researchers to investigate healthcare-related outcomes for individuals with major mental health disorders such as schizophrenia. ${ }^{2}$ The amalgamation and linkage of health record and genomic data-sets offers further potential and initial reports describing linkage are emerging. ${ }^{6,11-13}$ This type of approach has the potential to facilitate the collection of large-scale phenotypic data without the need to burden patients with extensive assessments. This may have particular advantage for physical health outcomes, which could be tracked longitudinally with updated health record linkage. 


\section{Study aims}

In this study, we aimed to link genetic data from a clinically ascertained sample of individuals with schizophrenia to anonymised NHS and administrative data-sets in the Secure Anonymised Information Linkage (SAIL) databank in Wales, ${ }^{9}$ with a focus on the rich physical health outcome data held in primary care electronic health resources. We then aimed to examine the association between physical health outcomes and genetic liability for schizophrenia as indexed by (a) rare (frequency $<1 \%$ ) CNVs, and (b) polygenic risk scores.

\section{Method}

\section{Participants}

Study individuals ( $n=958$, aged $17-84$ years, $41 \%$ female) from the Cardiff Cognition in Schizophrenia (CardiffCOGS) sample were recruited from community, in-patient and voluntary sector mental health services in the UK and underwent detailed phenotypic assessment including a Schedules for Clinical Assessment in Neuropsychiatry (SCAN) interview. ${ }^{14}$ Interview data and clinical case-note vignettes were then used to arrive at a best estimate lifetime diagnosis according to DSM-IV criteria. ${ }^{15}$ Ethical approval was obtained from relevant NHS multisite research ethics committees and written informed consent was obtained for all study participants for genetic research and linkage of their genetic information to the SAIL databank. Further information on the CardiffCOGS sample has been published elsewhere. ${ }^{16}$

\section{Electronic cohort from SAIL}

Data source and data linkage

The SAIL databank (http://www.saildatabank.com) is a national data repository containing anonymised, person-based, linkable data in Wales for over 3 million people. The procedure for linking research study data to SAIL has been described elsewhere. ${ }^{9,17,18}$ In brief, data from CardiffCOGS study individuals who had consented to linkage were imported into SAIL in line with permissions already granted to SAIL relating to good practice in research governance and privacy protection. We adopted a split file approach to separate individual identifiers from the interview data. Identity matching and creation of pseudonymised linkage keys (anonymised linking fields) were performed by a trusted third party prior to linkage and further encryption of data sets using deterministic matching based on NHS number or probabilistic matching using available demographics based on the Welsh Demographic Service data-set (all individuals registered with a general practice (GP) surgery). We included participants whose data were probabilistically linked with an adequate level of matching accuracy (matching score $\geq 0.9)$. $^{18}$

We used the General Practice Database (GPD), containing diagnoses, symptoms, investigations, prescribed medication, referrals, coded hospital contacts and test results. At time of analysis, 77\% (333/432) of GP surgeries in Wales supplied their data to SAIL. We also extracted data from the Patient Episode Database for Wales (PEDW), an NHS Wales hospital admissions data-set consisting of clinical information from all hospital admissions (inpatient and day cases) covering the entire population of Wales.

\section{Measures for health outcomes}

We used ICD-10 and Read codes for GDP and PEDW data-sets, respectively, to ascertain health outcomes. ${ }^{19}$ For schizophrenia, we adopted the codes that were validated and used in previous studies. ${ }^{18,20,21}$ We selected smoking, type 2 diabetes mellitus, ischaemic heart disease and body mass index (BMI) because of their high frequency in clinical samples of individuals with schizophrenia and their potential to contribute to increased mortality either directly, or via phenomena such as metabolic syndrome. We selected congenital disorders, intellectual disability and epilepsy as they are neurodevelopmental phenotypes with direct relevance to $\mathrm{CNV}$ carrier status. All of these variables also had either established SAIL algorithms or were considered to have high-quality data available in SAIL. We identified individuals with intellectual disability, ${ }^{22,23}$ ischaemic heart disease, ${ }^{17}$ epilepsy, ${ }^{24}$ diabetes mellitus ${ }^{25}$ and determined smoking status ${ }^{26}$ based on previously published works.

The list of codes used for extracting height, BMI and for identifying individuals with congenital disorders are given in supplementary Table 1 available at https://doi.org/10.1192/bjo.2020.42. For identifying schizophrenia, intellectual disability, ischaemic heart disease, epilepsy, diabetes mellitus and congenital disorders, we combined both the GPD and PEDW data-sets. We additionally extracted individuals' height, BMI and smoking status from the GPD where available.

For comparison, we extracted lifetime diagnoses and estimated crude rates, as well as age- and gender-standardised rates, of health outcomes for the whole population and those diagnosed with schizophrenia between the ages of 17 and 84 years (as at 30 June 2016). For estimating standardised rates from SAIL, crude rates were first computed for five age groups $(17-34,35-44,45-54,55-$ 64 and 65-84 years) for both genders. Standardised rates were then estimated based on the age and gender distribution in the clinical cohort. Linked data in SAIL were interrogated using structured query language (SQL DB2).

\section{Genetic data}

\section{Genotyping and CNV calling}

Samples were genotyped on OmniCombo or OmniExpress arrays (Illumina). ${ }^{16}$ After standard quality control, imputation was performed using IMPUTE $2^{27}$ and the 1000 genomes (phase 3) and UK10 K reference panels. ${ }^{28}$ Best-guess genotypes were generated with the following thresholds: minimal genotypic confidence $>90 \%$, INFO-score $>0.9$, minor allele frequency (MAF) $>1 \%$, and Hardy-Weinberg equilibrium $P$-value $<1 \times 10^{-10}$.

Full details of the CNV calling methods and quality controls measures used have been published elsewhere. ${ }^{29}$ Illumina Genome Studio (version 2011.1) was used to process raw intensity data into $\log \mathrm{R}$ ratios (LRR) and $\mathrm{B}$ allele frequencies. PennCNV (version 1.0.3.18) was then used to call CNVs based on 666868 probes common to all Illumina arrays used. ${ }^{30} \mathrm{CNVs}$ were joined if separated by less than $50 \%$ of their combined length. CNVs were excluded if they were (a) called with fewer than ten probes, (b) overlapped low copy repeats by more than $50 \%$ of their length, (c) had a probe density of less than 1 probe/20 kb, or (d) had a frequency of $>1 \%{ }^{31}$

\section{CNVS}

To examine for enrichment of rare, pathogenic CNVs in physical health comorbidity, we analysed the presence of $12 \mathrm{CNVs}$ robustly associated with the risk of schizophrenia, ${ }^{16,29}$ and $54 \mathrm{CNVs}$ nominally associated $(P<0.05)$ with intellectual disability, autism spectrum disorder or schizophrenia ('neurodevelopmental CNVs'). ${ }^{32}$ Following the approach taken in our previous work, 15q11.2 duplications were excluded because of their high frequency. ${ }^{33} \mathrm{CNV}$ burden analyses were carried out using PLINK on regions of variable copy number at two size thresholds (a) $\geq 500 \mathrm{~KB}$ and (b) $\geq 1 \mathrm{MB}$ and converted into carrier status for the purpose of regression analyses. 
Polygenic risk scores

Polygenic risk scores were calculated using the largest published schizophrenia genome-wide association study meta-analysis (39 915 individuals with schizophrenia, 64639 controls) as a training set and using the established method described by Wray et al. ${ }^{34,35}$ All study individuals were excluded from the training set. scores were generated using the -score function in PLINK ${ }^{31}$ for Single nucleotide polymorphisms with MAF $>10 \%$, INFO score $>0.9$, a low linkage disequilibrium to each other and excluding indels and the extended major histocompatibility complex region. Polygenic risk scores were calculated at nine $P$-value thresholds; $1 \times 10^{-8}, 1 \times 10^{-6}, 1 \times 10^{-4}, 1 \times 10^{-3}, 0.01,0.05,0.1,0.2$ and 0.5 .

\section{Analysis}

Rates of physical illness

Linked data in SAIL were interrogated using structured query language (SQL DB2). All crude rates and standardised rates of health outcomes were expressed as a percentage of population affected (lifetime prevalence). All standardised rate ratios (SRRs) and their $95 \%$ confidence intervals were calculated as previously described. $^{36-38}$

\section{Ascertainment rates of behaviours and diagnoses}

We evaluated the agreement on diagnoses between the clinical and electronic cohorts for each health outcome by constructing two $\times$ two contingency tables based on the paired responses from the interview and SAIL. Level of agreement was then assessed by unweighted Cohen's kappa coefficient ${ }^{39}$ and Gwet's AC1. ${ }^{40}$ The 95\% confidence intervals of Cohen's kappa and Gwet's AC1 were estimated as described in Fleiss, Cohen \& Everitt ${ }^{41}$ and Gwet, ${ }^{40}$ respectively. Strength of agreement metrics was categorised according to previously described criteria. ${ }^{42}$

\section{CNV analyses}

Association analyses were carried out using linear regression for average BMI (normalised using $\log _{10}$ transformation) and average height, and Firth logistic regression for all binary traits (type 2 diabetes mellitus, smoking, ischaemic heart disease, congenital disorders, epilepsy, intellectual disability). Firth logistic regression allows for the analysis of low numbers in addition to the inclusion of covariates. All analyses included age and gender as covariates. ${ }^{43}$

\section{Polygenic risk analyses}

We regressed a model for each polygenic risk score created from various training $P$-value thresholds against a base model including age, gender, the first five principal components and any additional principal components from the first 20 that were nominally associated with the phenotype of interest. We repeated these analyses with the addition of covariates reflecting symptom severity, nonresponse to antipsychotics, antipsychotic exposure, smoking status and genotyping platform (defined in supplementary Table 2). All statistical analyses were carried out in $\mathrm{R}$ and results were subject to Bonferroni correction for the eight phenotypes examined ( $P$-value threshold 0.0063).

\section{Results}

A total of 896 (93.5\%) study individuals from CardiffCOGS were linked to health records held in the SAIL databank. Linked study individuals had an age range of 17-84 years (mean 44 years), 371 $(41 \%)$ were female and $724(81 \%)$ had genetic data available.

\section{Physical health outcomes in CardiffCOGS and SAIL}

Table 1 and Fig. 1 outlines the frequencies of the physical health outcomes in the CardiffCOGS sample and SAIL. Within the SAIL population, individuals with schizophrenia had increased rates of epilepsy $(\mathrm{SRR}=5.34,95 \%$ CI 5.11-5.57), intellectual disability $(\mathrm{SRR}=3.11,95 \%$ CI 3.06-3.11), type 2 diabetes $(\mathrm{SRR}=2.45,95 \%$ CI 2.38-2.53), congenital disorders ( $\mathrm{SRR}=1.77$, 95\% CI 1.571.99), ischaemic heart disease ( $\mathrm{SRR}=1.57,95 \%$ CI $1.51-1.63)$ and smoking $(\mathrm{SRR}=1.44,95 \%$ CI 1.42-1.46). Individuals within CardiffCOGS had higher rates of type 2 diabetes (SRR $=1.29,95 \%$ CI 1.10-1.52) compared with the population with schizophrenia in SAIL. Crude unadjusted population rates are given in supplementary Table 3.

\section{Comparison of ascertainment rates of behaviours and diagnoses in CardiffCOGS and SAIL}

For physical health outcomes that were also reported at interview (smoking, type 2 diabetes mellitus, ischaemic heart disease, and epilepsy), we compared agreement with their health records (Table 2). Both Cohen's $\mathrm{k}$ and Gwet's AC1 for all physical conditions ranged from 0.502 to 0.936 . These show that the agreement of the rates ascertained from the interview and health records were moderate to high. ${ }^{42}$ The highest agreements were observed for ischaemic heart disease and epilepsy. The strength of agreement was lowest for smoking behaviour (Cohen's $\kappa=0.380$ and Gwet's $\mathrm{AC} 1=0.621$ ), reflecting only fair to substantial agreement.

\section{CNV}

A total of $2.1 \%(n=15)$ of the CardiffCOGS sample carried a schizophrenia-associated $\mathrm{CNV}, \quad 4.9 \% \quad(n=32) \quad$ carried a

\begin{tabular}{|c|c|c|c|c|c|}
\hline Phenotype & $\begin{array}{l}\text { CardiffCOGS } \\
\text { rate, } \%(n)\end{array}$ & $\begin{array}{l}\text { SAIL schizophrenia } \\
\text { population rate, } \%(n)\end{array}$ & $\begin{array}{l}\text { SAIL population } \\
\text { rate, } \%(n)\end{array}$ & $\mathrm{SRR}_{\text {sam, sch }}(95 \% \mathrm{Cl})$ & $\mathrm{SRR}_{\text {sch, gen }}(95 \% \mathrm{Cl})$ \\
\hline Congenital disorder & $1.34(12)$ & $0.93(326)$ & $0.53(21745)$ & $1.44(0.81-2.57)$ & 1.77 (1.57-1.99) \\
\hline Intellectual disability & $1.56(14)$ & $1.80(623)$ & $0.58(22142)$ & $0.87(0.51-1.48)$ & $3.11(3.06-3.11)$ \\
\hline Ischaemic heart disease & $6.93(62)$ & 6.99 (3449) & 4.46 (207 197) & $0.99(0.77-1.28)$ & $1.57(1.51-1.63)$ \\
\hline Epilepsy & $4.69(42)$ & 7.49 (2628) & $1.40(52020)$ & $0.63(0.46-0.85)$ & $5.34(5.11-5.57)$ \\
\hline Type 2 diabetes mellitus & $17.43(156)$ & 13.50 (5293) & $5.51(216$ 187) & $1.29(1.10-1.52)$ & $2.45(2.38-2.53)$ \\
\hline Smoking (current/ex) & $86.99(689)$ & 83.87 (22 120) & 58.38 (1 649 589) & $1.04(0.97-1.11)$ & $1.44(1.42-1.46)$ \\
\hline
\end{tabular}




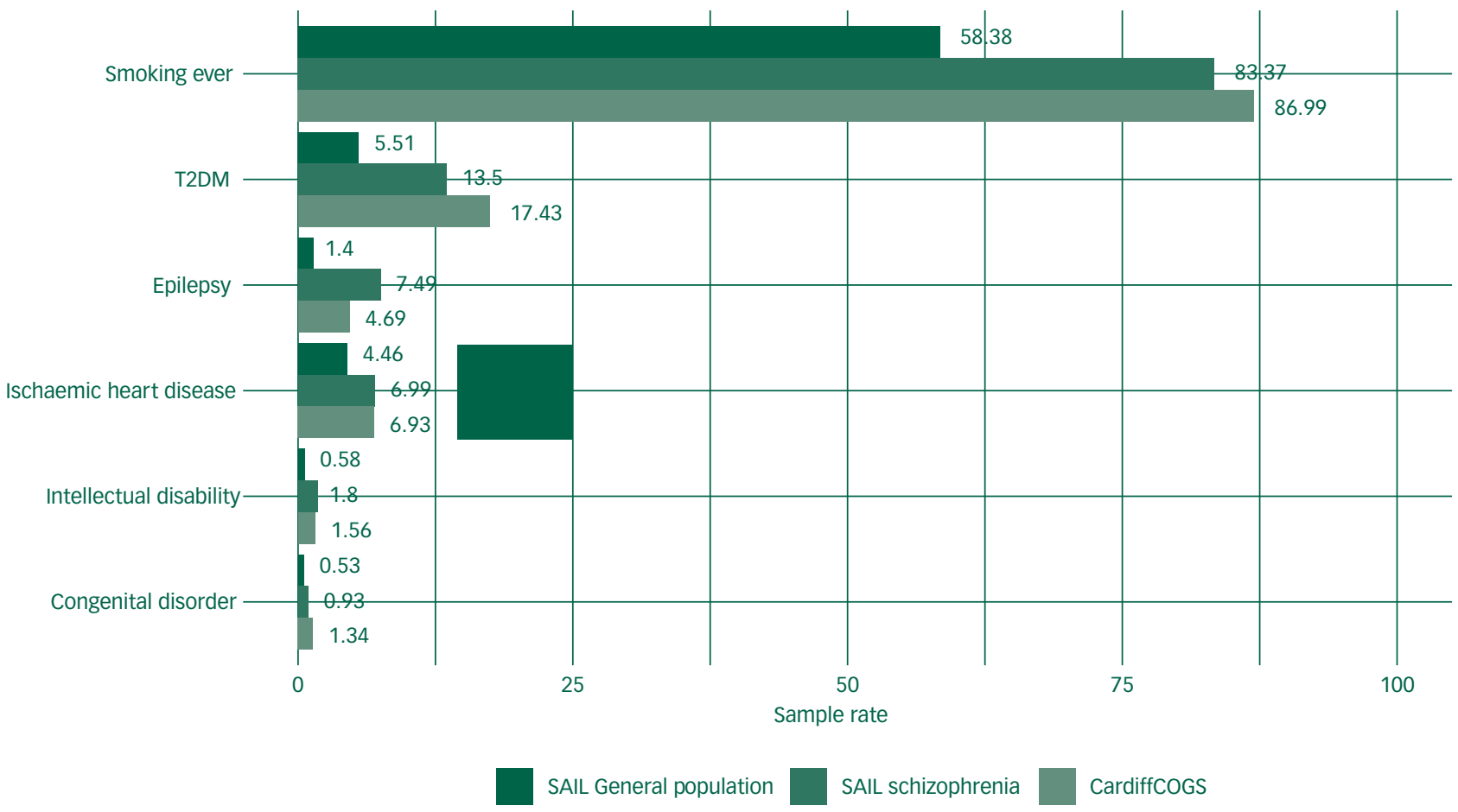

Fig. 1 Rates of physical health phenotypes in the Cardiff Cognition in Schizophrenia (CardiffCOG) schizophrenia sample, the schizophrenia population in Secure Anonymised Information Linkage (SAIL) and the general population in SAIL.

T2DM, type 2 diabetes mellitus.

neurodevelopmental $\mathrm{CNV}, 9.5 \%$ carried a chromosomal duplication or deletion $\geq 500 \mathrm{~KB}$ (19 deletions, 52 duplications, 2 both) and $3.2 \%$ carried a duplication or deletion $\geq 1 \mathrm{MB}$ (6 deletions, 17 duplications). We found no evidence that CNV carriers had increased rates of poor physical health outcomes (Table 3). However, average height was nominally associated with carrier status for CNVs associated with schizophrenia (beta $=-0.075$, $95 \% \mathrm{CI}-0.14$ to $-0.01, P=0.017)$ and neurodevelopmental disorders (beta $=-0.077,95 \% \mathrm{CI}-0.64$ to $-0.07, P=0.015$ ) (Table 3 ). $\mathrm{CNV}$ carriers were on average $7.5-7.7 \mathrm{~cm}$ shorter than non-carriers. There was no evidence for association between rare CNVs of $500 \mathrm{~KB}$ or greater and any of the phenotypes examined (supplementary Table 4).

\section{Polygenic risk for schizophrenia}

We found no evidence for an association between polygenic risk scores for schizophrenia and the physical health outcomes studied (Fig. 2, supplementary Table 5), although there was weak evidence for an association with ischaemic heart disease at the genome-wide $P$-value threshold (odds ratio $(\mathrm{OR})=1.65$, $95 \%$ CI $1.22-2.24$; adjusted $R^{2}=0.035, P=0.001$ ). The lack of

Table 2 Interrater agreement of the rates of ascertainment between the Cardiff Cognition in Schizophrenia (CardiffCOG) interview and linkage to

National Health Service records ${ }^{\mathrm{a}}$

\begin{tabular}{|c|c|c|c|c|c|c|}
\hline \multirow[b]{2}{*}{ SAIL } & \multicolumn{2}{|c|}{$\begin{array}{l}\text { CardiffCOGS } \\
\text { interview }\end{array}$} & \multirow{2}{*}{$\begin{array}{l}\text { \% Reported affected } \\
\text { at CardiffCOGS } \\
\text { interview }\end{array}$} & \multirow{2}{*}{$\begin{array}{c}\% \text { Reported } \\
\text { affected in SAIL }\end{array}$} & \multirow[b]{2}{*}{ Cohen's к $(95 \% \mathrm{Cl})$} & \multirow[b]{2}{*}{ Gwet's AC1 $(95 \% \mathrm{Cl})$} \\
\hline & Yes & No & & & & \\
\hline \multicolumn{7}{|l|}{ T2DM } \\
\hline Affected & 75 & 43 & 13.34 & 17.69 & $0.675(0.597-0.754)$ & $0.884(0.853-0.915)$ \\
\hline Unaffected & 14 & 535 & & & & \\
\hline \multicolumn{7}{|c|}{ Ischaemic heart disease } \\
\hline Affected & 21 & 19 & 5.96 & 6.12 & $0.502(0.362-0.641)$ & $0.936(0.915-0.957)$ \\
\hline Unaffected & 18 & 596 & & & & \\
\hline Affected & 24 & 10 & 7.81 & 5.11 & $0.529(0.397-0.661)$ & $0.935(0.914-0.956)$ \\
\hline Unaffected & 28 & 604 & & & & \\
\hline \multicolumn{7}{|l|}{ Smoking } \\
\hline Affected & 515 & 119 & 71.92 & 77.41 & $0.380(0.307-0.452)$ & $0.621(0.567-0.675)$ \\
\hline Unaffected & 74 & 111 & & & & \\
\hline $\begin{array}{l}\text { SAlL, Secure } A \\
\text { a. The smokin } \\
\text { first order agre } \\
\text { moderate agre }\end{array}$ & d infor & inkage & $\begin{array}{l}\text { veen o (chance, or no agr } \\
\text { tantial agreement. }\end{array}$ & $\begin{array}{l}\text { ar smoker at interviev } \\
11 \text { (perfect agreemen }\end{array}$ & $\begin{array}{l}\text { refers to Cohen's kappas } \\
\text { low } 0.4 \text { indicate poor agre }\end{array}$ & $\begin{array}{l}{ }^{15} \text { Gwet's AC1 refers to the } \\
\text { values of } 0.4-0.59 \text { indicate }\end{array}$ \\
\hline
\end{tabular}


Table 3 Association analysis results for physical health phenotypes and two groups of copy number variants (CNVs, 12 schizophrenia CNVs and 53 neurodevelopmental CNVS) ${ }^{a}$

\begin{tabular}{|c|c|c|c|c|c|c|c|c|}
\hline \multirow[t]{2}{*}{ Phenotype } & \multicolumn{4}{|c|}{ Schizophrenia CNVs } & \multicolumn{4}{|c|}{ Neurodevelopmental CNVs } \\
\hline & Carriers & $\begin{array}{c}\text { Non- } \\
\text { carriers }\end{array}$ & Effect size $(95 \% \mathrm{Cl})$ & $P$ & Carriers & $\begin{array}{c}\text { Non- } \\
\text { carriers }\end{array}$ & Effect size $(95 \% \mathrm{Cl})$ & $P$ \\
\hline Average height (GP) & 13 & 611 & $-0.075(-0.14$ to -0.01$)$ & 0.017 & 31 & 593 & $-0.077(-0.64$ to -0.07$)$ & 0.015 \\
\hline Average BMl $\log _{10}(\mathrm{GP})$ & 13 & 604 & $0.04(-0.03$ to 0.09$)$ & 0.32 & 30 & 587 & $0.02(-0.26$ to 0.48$)$ & 0.56 \\
\hline \multicolumn{9}{|c|}{ Type 2 diabetes mellitus (both) } \\
\hline Affected & $<5$ & 126 & $0.45(-0.79$ to 1.53$)$ & 0.45 & 6 & 124 & $-0.15(-1.12$ to 0.68$)$ & 0.74 \\
\hline Unaffected & 11 & 576 & & & 30 & 557 & & \\
\hline \multicolumn{9}{|c|}{ Smoking - current or ex-smoker (GP) } \\
\hline Affected & 9 & 552 & $-0.85(-1.86$ to 0.21$)$ & 0.11 & 25 & 536 & $-0.46(-1.15$ to 0.29$)$ & 0.23 \\
\hline Unaffected & $<5$ & 78 & & & 7 & 75 & & \\
\hline \multicolumn{9}{|c|}{ Ischaemic heart disease (both) } \\
\hline Affected & 0 & 53 & $-1.15(-6.03$ to 0.99$)$ & 0.36 & $<5$ & 52 & $-1.08(-3.31$ to 0.35$)$ & 0.16 \\
\hline Unaffected & 15 & 656 & & & 35 & 636 & & \\
\hline \multicolumn{9}{|c|}{ Congenital disorders (both) } \\
\hline Affected & $<5$ & 8 & $1.97(-0.32$ to 3.59$)$ & 0.08 & $<5$ & 8 & $1.29(-0.97$ to 2.83$)$ & 0.21 \\
\hline Unaffected & 14 & 701 & & & 35 & 680 & & \\
\hline \multicolumn{9}{|l|}{ Epilepsy (both) } \\
\hline Affected & $<5$ & 36 & $1.23(-0.43$ to 2.48$)$ & 0.13 & $<5$ & 34 & $0.94(-0.24$ to 1.89$)$ & 0.11 \\
\hline Unaffected & 13 & 673 & & & 32 & 654 & & \\
\hline \multicolumn{9}{|c|}{ Intellectual disability (both) } \\
\hline Affected & $<5$ & 8 & $2.13(-0.17$ to 3.73$)$ & 0.06 & $<5$ & 8 & $1.26(-0.99$ to 2.79$)$ & 0.22 \\
\hline Unaffected & 14 & 701 & & & 5 & 680 & & \\
\hline
\end{tabular}

association between schizophrenia polygenic risk scores and physical health outcomes remained in sensitivity analyses covarying for symptom severity, non-response to antipsychotics, antipsychotic exposure, smoking status and genotyping array (supplementary Table 6). However, we did identify significant associations between non-response to antipsychotics and type 2 diabetes $(\mathrm{OR}=2.94,95 \% \mathrm{CI} 1.79-4.85, P \leq 0.0001)$ and an association between symptom severity and intellectual disability $(\mathrm{OR}=1.24, \quad 95 \% \quad \mathrm{CI} \quad 1.05-1.46, \quad P=0.0012)$. There were additional nominal associations $(P<0.05)$ between non-response to antipsychotics and epilepsy, and smoking and ischaemic heart disease (supplementary Table 6).

\section{Discussion}

In this study, we report the linkage of genetic data from a clinically ascertained sample of individuals with schizophrenia to (a)

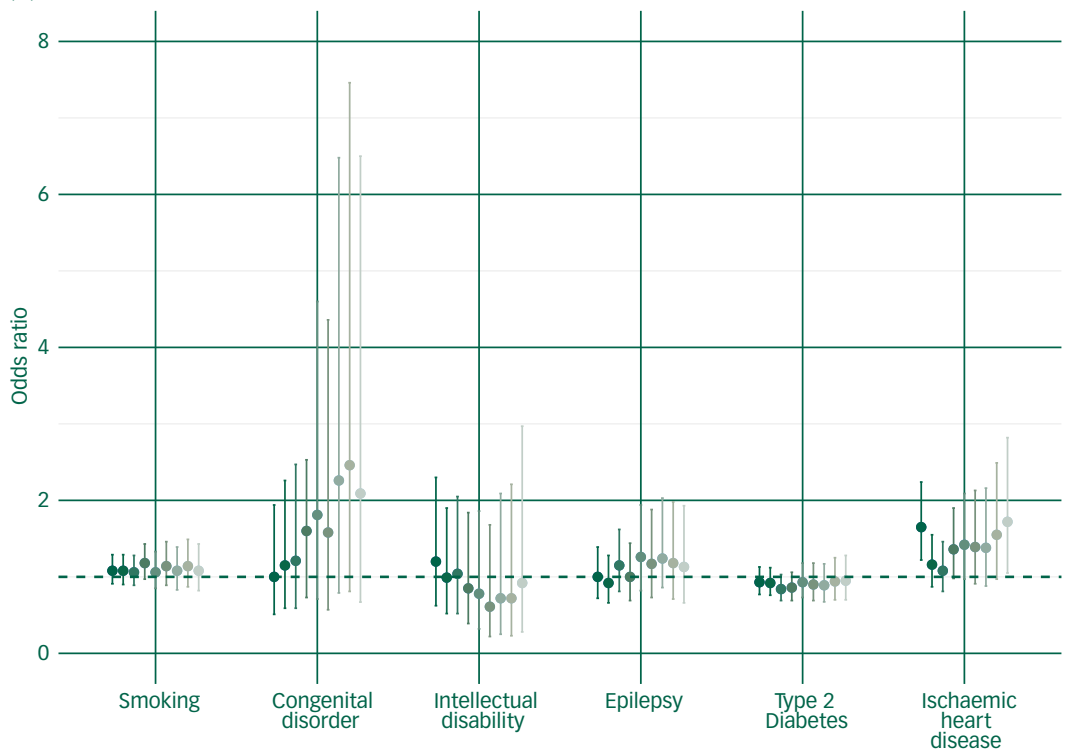

(b)

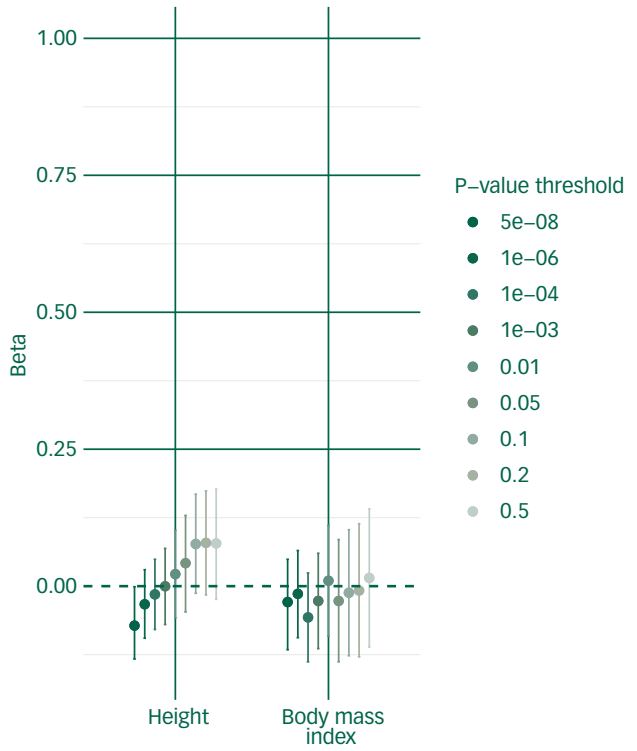

Fig. 2 Graphs of the results from regression models for the association between polygenic risk for schizophrenia and physical health outcomes.

(a) Odds ratio; (b) Beta. Odds ratios are shown for the $P$-value thresholds at which markers were selected. 
anonymised NHS health records in the SAIL databank. Consistent with data collected at interview, we found that individuals with schizophrenia in Wales had increased rates of neurodevelopmental disorders (epilepsy, intellectual disability and congenital disorders), smoking, type 2 diabetes mellitus and ischaemic heart disease compared with the general population. However, the results of our genetic analyses suggest that these increased rates are not because of genetic liability to schizophrenia; we found no evidence for an association between genetic risk for schizophrenia indexed by $\mathrm{CNVs}$ or polygenic risk scores and physical health outcomes.

These findings are supported by a recent population-based study that linked genetic and health record data for 106160 individuals and found an inverse association between increased schizophrenia polygenic risk scores and obesity and an inverse association with diabetes when controlling for schizophrenia diagnosis or for antipsychotic medication history. ${ }^{6}$ No association was found between schizophrenia polygenic risk scores and the other physical health outcomes found in this study. ${ }^{6}$ Thus, the evidence available indicates that increased rates of poor physical health observed in patients with schizophrenia is unlikely to be driven by the genetic liability for the disorder.

There may be many other factors contributing to an increased rate of the physical health outcomes examined. For the non-neurodevelopmental outcomes, these include, but are not limited to, medication side-effects and lifestyle choices such as smoking and diet. For example, weight gain caused by antipsychotic medication, poor diet and smoking may all contribute to the risk of type 2 diabetes mellitus and ischaemic heart disease. This is supported by our study; we found significant associations between non-response to antipsychotics and type 2 diabetes mellitus and nominal associations between non-response to antipsychotics and epilepsy, and smoking and ischaemic heart disease. The associations with nonresponse to antipsychotics may reflect the frequent use of clozapine in this patient group, an antipsychotic known to cause weight gain and increase the risk of type 2 diabetes mellitus. Our additional analyses also found that intellectual disability was significantly associated with a greater symptom severity. The reasons for this association are not yet clear. Importantly, several of these nongenetic factors are amenable to targeted intervention, which may reduce the risk of their consequent physical health outcomes.

Our finding that carriers of rare neurodevelopmental CNVs tend to be shorter than $\mathrm{CNV}$ non-carriers is in keeping with previous work on CNV associations with anthropometric traits in a sample of 191 161 adults. ${ }^{44}$ Macé et al report associations between both total CNV burden, several individual CNV loci (also examined in our study) and alterations in height. Additional findings from Macé et al of a lack of association between anthropomorphic traits and schizophrenia suggest that the CNVs affect height independent of disease. ${ }^{44}$

The rates of smoking ascertained in our study (87.0\%) and in those with schizophrenia in SAIL (83.9\%) appear high but are comparable with several studies also examining 'ever' smoking in individuals with schizophrenia. ${ }^{45}$ Similarly, the rates of ischaemic heart disease in the CardiffCOGS and SAIL schizophrenia population groups (both 6.9\%) are comparable with rates established in other studies.43,44 CardiffCOGS had a higher rate of type 2 diabetes mellitus (17.4\%) than the schizophrenia population in SAIL (13.5\%) and other study samples such as a Swedish cohort study of individuals with schizophrenia (11-12.5\%). ${ }^{1}$ This may reflect the higher than average proportion of individuals with chronic schizophrenia and long-term antipsychotic medication use in the CardiffCOGS sample.

\section{Study limitations}

The main limitation of this study was related to sample size; it is possible that we were underpowered to detect genetic associations with small effect sizes. However, it can still be concluded that genetic liability to schizophrenia does not have a large or significant impact on the occurrence of physical comorbidity. Nonetheless, our plan for future work is to link genetic data for a far greater number of individuals to their health records. This study does provide an important exemplar for the value of linking genetic data to routinely collected health-related data. Such an approach has great potential to generate a wealth of evidence, which can be translated into improved health outcomes for patients.

Kimberley M. Kendall, BSC, MBBCh, MRCPsych, MRC Centre for Neuropsychiatric Genetics and Genomics, Division of Psychological Medicine and Clinical Neurosciences, Cardiff University, UK' Ann John (D, BSC, MBBS, MRCGP, MPH, FFPH, PGCHE, Health Data Research UK, Swansea University Medical School, Swansea University, UK; Sze Chim Lee, BEng, BA, MSc, PhD, Health Data Research UK, Swansea University Medical School, Swansea University, UK; Elliott Rees, BSC, MSC, PhD, MRC Centre for

Neuropsychiatric Genetics and Genomics, Division of Psychological Medicine and Clinical Neurosciences, Cardiff University, UK; Antonio F. Pardiñas, BSC, MSc, PhD, MRC Centre for Neuropsychiatric Genetics and Genomics, Division of Psychological Medicine and Clinical Neurosciences, Cardiff University, UK; Marcos Del Pozo Banos, BSC, MSC, PhD, Health Data Research UK, Swansea University Medical School, Swansea University, UK; Michael J. Owen, BSC, MB, ChB, PhD, FRCPsych, MRC Centre for Neuropsychiatric Genetics and Genomics, Division of Psychological Medicine and Clinical Neurosciences, Cardiff University, UK; Michael C. O'Donovan, BSC, MB, PhD, FRCPsych, MRC Centre for Neuropsychiatric Genetics and Genomics, Division of Psychological Medicine and Clinical Neurosciences, Cardiff University, UK : George Kirov $\mathbb{D}$, MD, PhD, MRCPsych, MRC Centre for Neuropsychiatric Genetics and Genomics, Division of Psychological Medicin and Clinical Neurosciences, Cardiff University, UK; Keith Lloyd, MBBS, MSc(econ), MSC, MD, FRCP(Edin) FRCPsych, FRCPsych, Health Data Research UK, Swansea University Medical School, Swansea University, UK; Ian Jones, BSC, MBBS, MSC, PhD, MRCPsych, National Centre for Mental Health, MRC Centre for Neuropsychiatric Genetics and Genomics, Division of Psychological Medicine and Clinical Neurosciences, Cardiff University, UK; Sophie E. Legge (1D, BSC, PhD, MRC Centre for Neuropsychiatric Genetics and Genomics, Division of Psychological Medicine and Clinical Neurosciences, Cardiff University, UK; James T. R. Walters, BM, MSC, PhD, MRCPsych, MRC Centre for Neuropsychiatric Genetics and Genomics, Division of Psychological Medicine and Clinical Neurosciences, Cardiff University, UK

Correspondence: James Walters. Email: waltersjt@cardiff.ac.uk

First received 4 Nov 2019, final revision 2 Apr 2020, accepted 28 Apr 2020

\section{Supplementary material}

Supplementary material is available online at https://doi.org/10.1192/bjo.2020.42

\section{Data availability}

All data requests should be submitted to the corresponding authors for consideration.

\section{Acknowledgements}

We thank the participants, clinicians, field team and MRC Centre for Neuropsychiatric Genetics and Genomics laboratory staff for their help with the CardiffCOGS study. We thank the staff at deCODE Genetics for sample genotyping.

\section{Author contributions}

K.K., A.J., S.C.L., S.E.L. and J.T.R.W. made substantial contributions to the conception and design of the study. All authors contributed either to the acquisition or analysis of the data. K.K., S.C.L. and S.L. carried out the data analysis. K.K., A.J., S.C.L., S.E.L. and J.T.R.W. drafted the manuscript. All authors contributed to the critical revision of the manuscript for important intellectual conAll authors contributed to the critical revision of the manuscri
tent and gave final approval of the version to be published.

\section{Funding}

This project was funded by a Wellcome Trust Institutional Strategic Support Fund (ISSF) Populations Pilot Award. This project was supported also by the following grants: Medical Research Council Centre (MR/L010305/1). Program (G0800509) and Project (MC PC 17212) grants to Cardiff University, Medical Research Council Pathfinder (MR_PC 17211) to Swansea University, an MQ grant (MQDS16/36) to Cardiff University and the Health and Care Research Wales National Centre for Mental Health (155838) at Swansea University. K.K. was funded by a Wellcome Trust Clinical Research Training Fellowship.

\section{Declaration of interest}

The authors declare no conflicts of interest. 


\section{References}

1 Crump C, Winkleby MA, Sundquist K, Sundquist J. Comorbidities and mortality in persons with schizophrenia: a Swedish national cohort study. Am J Psychiatry 2013; 170: 324-33.

2 Smith DJ, Langan J, McLean G, Guthrie B, Mercer SW. Schizophrenia is associated with excess multiple physical-health comorbidities but low levels of recorded cardiovascular disease in primary care: cross-sectional study. BMJ Open 2013: 3: e002808.

3 National Institute for Health and Care Excellence. Schizophrenia: Core Interventions in the Treatment and Management of Schizophrenia in Adults in Primary and Secondary Care. National Institute for Health and Care Excellence, 2009.

4 Crawford K, Bracher-Smith M, Kendall KM, Rees E, Pardinas AF, Einon M, et al Medical consequences of pathogenic CNVs in adults: analysis of the UK Biobank. J Med Genet 2019; 56: 131-8.

5 Owen D, Bracher-Smith M, Kendall K, Rees E, Einon M, Escott-Price V, et al. Effects of pathogenic CNVs on physical traits in participants of the UK Biobank. BMC Genomics 2018; 19: 867.

6 Zheutlin $A B$, Dennis J, Linnér R, Moscati $A$, Restrepo $N$, Straub $P$, et al. Penetrance and pleiotropy of polygenic risk scores for schizophrenia in 106,160 patients across four health care systems. Am J Psychiatry 2019; 176 (10): 846-55.

7 Cederlöf M, Bergen SE, Larsson H, Landén M, Lichtenstein P. Acute intermittent porphyria: comorbidity and shared familial risks with schizophrenia and bipolar disorder in Sweden. Br J Psychiatry 2015; 207: 556-7.

8 Cesta CE, Månsson M, Palm C, Lichtenstein P, lliadou AN, Landén M. Polycystic ovary syndrome and psychiatric disorders: co-morbidity and heritability in a nationwide Swedish cohort. Psychoneuroendocrinology 2016; 73: 196-203.

9 Lyons RA, Jones KH, John G, Brooks CJ, Verplancke JP, Ford DV, et al. The SAIL databank: linking multiple health and social care datasets. BMC Med Inf Decis Mak 2009; 9: 3.

10 Stewart R, Soremekun M, Perera G, Broadbent M, Callard F, Denis M, et al. The South London and Maudsley NHS Foundation Trust Biomedical Research Centre (SLAM BRC) case register: development and descriptive data. BMC Psychiatry 2009; 9: 51.

11 Kerr SM, Campbell A, Marten J, Vitart V, McIntosh AM, Porteous DJ, et al. Electronic health record and genome-wide genetic data in Generation Scotland participants. Wellcome Open Res 2017; 2: 85

12 Stahl EA, Breen G, Forstner AJ, McQuillin A, Ripke S, Trubetskoy V, et al. Genome-wide association study identifies 30 loci associated with bipolar disorder. Nat Genet 2019; 51: 793-803.

13 Crawford DC, Crosslin DR, Tromp G, Kullo IJ, Kuivaniemi $\mathrm{H}$, Hayes MG, et al. eMERGEing progress in genomics-the first seven years. Front Genet 2014; 5: 184

14 Wing JK, Babor T, Brugha T, Burke J, Cooper JE, Giel R, et al. SCAN. Schedules for Clinical Assessment in Neuropsychiatry. Arch Gen Psychiatry 1990; 47 589-93.

15 American Psychiatric Association. Diagnostic and statistical manual of mental disorders 4th edn, 2000

16 Rees E, Walters JT, Georgieva L, Isles AR, Chambert KD, Richards AL, et al. Analysis of copy number variations at 15 schizophrenia-associated loci. $\mathrm{Br} \mathrm{J}$ Psychiatry 2014; 204: 108-14.

17 Rapsomaniki E, Timmis A, George J, Pujades-Rodriguez M, Shah AD, Denaxas S, et al. Blood pressure and incidence of twelve cardiovascular diseases: lifetime risks, healthy life-years lost, and age-specific associations in 1.25 million people. Lancet 2014; 383: 1899-911.

18 Lloyd K, McGregor J, John A, Craddock N, Walters JT, Linden D, et al. A national population-based e-cohort of people with psychosis (PsyCymru) linking prospectively ascertained phenotypically rich and genetic data to routinely collected records: overview, recruitment and linkage. Schizophr Res 2015; 166 $131-6$

19 World Health Organization. The ICD-10 classification of mental and behavioural disorders: Clinical descriptions and diagnostic guidelines. World Health Organization, 1992.

20 John A, McGregor J, Jones I, Lee SC, Walters JTR, Owen MJ, et al. Premature mortality among people with severe mental illness - new evidence from linked primary care data. Schizophr Res 2018; 199: 154-62.

21 Economou A, Grey M, McGregor J, Craddock N, Lyons RA, Owen MJ, et al. The health informatics cohort enhancement project (HICE): using routinely collected primary care data to identify people with a lifetime diagnosis of psychotic disorder. BMC Res Notes 2012; 5: 95.

22 Rahman MA, Todd C, John A, Tan J, Kerr M, Potter R, et al. School achievement as a predictor of depression and self-harm in adolescence: linked education and health record study. Br J Psychiatry. 2018; 212: 215-221.
23 Brophy S, Kennedy J, Fernandez-Gutierrez F, John A, Potter R, Linehan C, et al. Characteristics of children prescribed antipsychotics: analysis of routinely collected data. J Child Adolesc Psychopharmacol. 2018; 28(3): 180-191.

24 Pickrell WO, Lacey AS, Bodger OG, Demmler JC, Thomas RH, Lyons RA, et al. Epilepsy and deprivation, a data linkage study. Epilepsia 2015; 56: 585-91.

25 Eastwood SV, Mathur R, Atkinson M, Brophy S, Sudlow C, Flaig R, et al. Algorithms for the capture and adjudication of prevalent and incident diabetes in UK Biobank. PLoS One 2016; 11: e0162388.

26 Atkinson MD, Kennedy Jl, John A, Lewis KE, Lyons RA, Brophy ST, et al. Development of an algorithm for determining smoking status and behaviour over the life course from UK electronic primary care records. BMC Med Inf Decis Mak 2017; 17: 2

27 Howie BN, Donnelly P, Marchini J. A flexible and accurate genotype imputation method for the next generation of genome-wide association studies. PLOS Genet 2009; 5: e1000529.

28 Huang J, Howie B, McCarthy S, Memari Y, Walter K, Min JL, et al. Improved imputation of low-frequency and rare variants using the UK10K haplotype reference panel. Nat Commun 2015; 6: 8111.

29 Rees E, Kendall K, Pardiñas AFF, Legge SEE, Pocklington A, Escott-Price V, et al. Analysis of intellectual disability copy number variants for association with schizophrenia. JAMA Psychiatry 2016; 73: 963-9.

30 Wang K, Li M, Hadley D, Liu R, Glessner J, Grant SF, et al. PennCNV: an integrated hidden Markov model designed for high-resolution copy number variation detection in whole-genome SNP genotyping data. Genome Res 2007; 17 1665-74.

31 Purcell S, Neale B, Todd-Brown K, Thomas L, Ferreira MA, Bender D, et al. PLINK a tool set for whole-genome association and population-based linkage analyses. Am J Hum Genet 2007; 81: 559-75.

32 Coe BP, Witherspoon K, Rosenfeld JA, van Bon BW, Vulto-van Silfhout AT, Bosco $P$, et al. Refining analyses of copy number variation identifies specific genes associated with developmental delay. Nat Genet 2014; 46: 1063-71.

33 Kendall KM, Rees E, Escott-Price V, Einon M, Thomas R, Hewitt J, et al. Cognitive performance among carriers of pathogenic copy number variants: analysis of 152,000 UK Biobank subjects. Biol Psychiatry 2017; 82: 103-10.

34 Pardiñas AF, Holmans P, Pocklington AJ, Escott-Price V, Ripke S, Carrera N, et al. Common schizophrenia alleles are enriched in mutation-intolerant genes and in regions under strong background selection. Nat Genet 2018; 50: 381-9

35 Wray NR, Lee SH, Mehta D, Vinkhuyzen AAE, Dudbridge F, Middeldorp CM. Research review: polygenic methods and their application to psychiatric traits. 2014; 55: 1068-87.

36 Armitage P, Berry G, Matthews J. Statistical methods in medical research. In Statistical Methods in Medical Research (4th edn). John Wiley and Sons, 2002.

37 Flanders WD. Approximate variance formulas for standardized rate ratios. J Chronic Dis 1984; 37 : 449-53.

38 Newman S.Biostatistical Methods in Epidemiology. John Wiley and Sons, 2002.

39 Cohen J. A coefficient of agreement for nominal scales. Educ Psychol Meas 1960; 20: 37-46.

40 Gwet KL. Computing inter-rater reliability and its variance in the presence of high agreement. Br J Math Stat Psychol 2008; 61: 29-48.

41 Fleiss J, Cohen J, Everitt B. Large sample standard errors of kappa and weighted kappa. Psychol Bull 1969; 72: 323-7.

42 Landis JR, Koch GG. The measurement of observer agreement for categorical data. Biometrics 1977; 33: 159-74.

43 Wang $X$. Firth logistic regression for rare variant association tests. Front Genet 2014; 5: 187

44 Mace A, Tuke MA, Deelen P Kristiansson K, Mattsson H, Nõukas M, et al. 2017. CNV-association meta-analysis in 191,161 European adults reveals new loci associated with anthropometric traits. Nat Commun 2017; 8: 744.

45 de Leon J, Diaz FJ. A meta-analysis of worldwide studies demonstrates an association between schizophrenia and tobacco smoking behaviors. Schizophr Res 2005; 76: 135-57.

46 Ford DV, Jones KH, Verplancke JP, Lyons RA, John G, Brown G, et al. The SAIL Databank: building a national architecture for e-health research and evaluation. BMC Health Serv Res 2009; 9: 157.

47 Goff DC, Sullivan LM, McEvoy JP, Meyer JM, Nasrallah HA, Daumit GL, et al. A comparison of ten-year cardiac risk estimates in schizophrenia patients from the CATIE study and matched controls. Schizophr Res 2005 Dec; 80: 45-53.

48 Cohn T, Prud'homme D, Streiner D, Kameh H, Remington G. Characterizing coronary heart disease risk in chronic schizophrenia: high prevalence of the metabolic syndrome. Can J Psychiatry 2004 Nov; 49: 753-60.
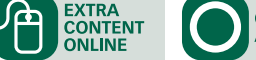
OPEN
ACCESS 